\title{
Ruxolitinib as an emerging treatment in myelofibrosis
}

\section{Robyn M Emanuel' \\ Holly L Geyer' \\ Ruben A Mesa ${ }^{2}$}

'Department of Internal Medicine, ${ }^{2}$ Department of Hematology and Oncology, Mayo Clinic, Scottsdale, AZ, USA

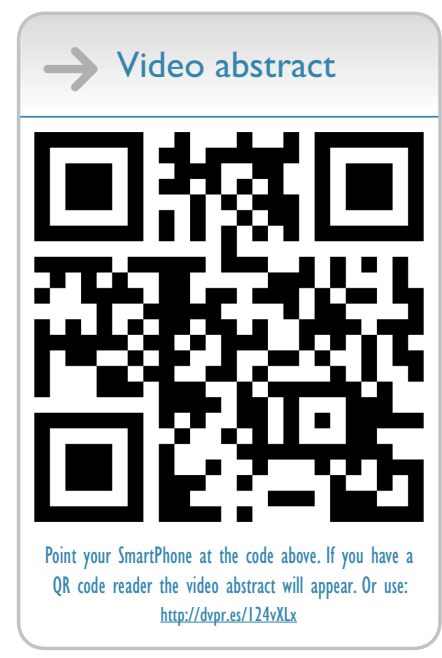

Correspondence: Ruben A Mesa Division of Hematology and Oncology, Mayo Clinic, I3400 East Shea Boulevard, Scottsdale, AZ, USA

Tel +l 48030 I 4502

Fax + I 48030 I 4675

Email mesa.ruben@mayo.edu

\author{
This article was published in the following Dove Press journal: \\ Blood and Lymphatic Cancer:Targets and Therapy \\ 8 March 2013 \\ Number of times this article has been viewed
}

\begin{abstract}
Essential thrombocythemia, polycythemia vera, and myelofibrosis belong to the class of Bcr-Abl negative hematologic neoplasms, which arise in part from varying Janus kinase-2 (JAK2) cellular deregulation. With the development of novel tyrosine kinase inhibitors capable of successfully inhibiting JAK in vivo, an influx of JAK2 inhibitors has come under clinical investigation. Ruxolitinib (Jakafi ${ }^{\circledR}$; Incyte Corporation, Wilmington, DE, USA) was the first of these compounds to gain US Food and Drug Administration approval in late 2011 for the treatment of intermediate- and high-risk myelofibrosis. Two Phase III clinical trials - Controlled Myelofibrosis Study with Oral JAK Inhibitor Treatment-I and -II (COMFORT-I and -II) - played key roles in the US Food and Drug Administration approval of ruxolitinib with successful demonstration of spleen reduction and symptom palliation. Well tolerated in most patients, common side effects include cytopenias and gastrointestinal toxicities. The majority of preliminary data appears to suggest that if administered in a dose-titrated fashion, ruxolitinib can be used safely in a clinical practice setting. Additionally, patients most likely to benefit from ruxolitinib treatment are those with moderate to severe constitutional symptoms or splenomegaly. Future studies are ongoing in applying ruxolitinib to other hematologic and solid tumor malignancies. More clinical experience is recommended before the utility of this medication in a routine clinical practice setting can be fully determined.
\end{abstract}

Keywords: myeloproliferative neoplasms, myelofibrosis, ruxolitinib, JAK2 inhibitors, INCB018424

\section{Introduction}

First designated as a disease class in 1951 by Sir William Dameshek, the classical Bcr-Abl negative myeloproliferative neoplasms (MPNs) include polycythemia vera (PV), essential thrombocythemia (ET), and myelofibrosis (MF). MF can arise as a primary event (primary MF [PMF]), or can arise from antecedent PV (post-PV MF) or ET (post-ET MF). Arising individually from genetic and epigenetic changes to the DNA structures of the hematopoietic cell, each MPN subtype is associated with a variable presentation, disease course, and prognosis. The nuances to each disease subtype's diagnosis, prognosis, and optimal treatment have only recently been brought to light with the discovery of the Janus kinase-2 (JAK2) mutation, JAK2 $2^{V 617 F}$, in 2004 and the subsequent publication of this finding in 2005. ${ }^{1}$ This revolutionary discovery became the first clue to the specific disease mechanism and concomitantly aided in the development of effective molecular targets. In this new age of gene-targeted therapies, JAK2 inhibitors have displayed particular effectiveness in decreasing splenomegaly and resolving constitutional symptoms with minimal 
myelosuppression and drug-related toxicities. Moreover, recent data suggests that certain JAK2 inhibitors may even offer a therapeutic survival advantage. Ruxolitinib (Jakaf ${ }^{\circledR}$; Incyte Corporation, Wilmington, DE, USA) was the first of these unique therapies to receive US Food and Drug Administration approval in November 2011. This review will discuss the disease pathophysiology, molecular genetics, benefits, toxicities, and ongoing applications of this innovative therapy.

Mutation of $J A K 2^{V 617 F}$ is present within $55 \%$ of ET patients, $96 \%$ of $\mathrm{PV}$, and $65 \%$ of PMF patients. These MPNs have been found to occur at an incidence of approximately six per 100,000 individuals and are commonly diagnosed beyond the fifth decade of life. ${ }^{2}$ MPN life expectancy varies by subtype. Studies of over 2000 patients with PV and ET indicate that ET has a life expectancy similar to age-matched controls. ${ }^{3} \mathrm{PV}$ patients have an expectancy of approximately 19 years. ${ }^{4}$ For patients with PV or ET, progression into post-ET or post-PV MF and later transformation to acute leukemia occurred at a rate of approximately $0.7 \%$ and $2.5 \%$ over a period of 10 years, respectively. ${ }^{5} \mathrm{MF}$ carries the worst prognosis among the Bcr-Abl negative MPNs, with a life expectancy of only 4-7 years and a mean age at diagnosis of 65 years. ${ }^{6,7}$ For patients with MF, primary or secondary transformation into acute myeloid leukemia is common, occurring at a rate of approximately $9 \%$ over a period of 5 years for patients with PMF. ${ }^{8}$

\section{MPN prognostic scoring}

Due to challenges with estimating prognosis in PMF, three main prognostic scoring systems are available (Table 1). PMF prognosis can be scored at the time of diagnosis using the International Working Group Prognostic Scoring System (IPSS). IPSS scoring utilizes the factors of age ( $>65$ years), blasts present in peripheral blood ( $\geq 1 \%$ ), hemoglobin $(<10 \mathrm{~g} / \mathrm{dL})$, leukocyte count $\left(>25 \times 10^{9} / \mathrm{L}\right)$, and the presence of constitutional symptoms (including weight loss $>10 \%$, night sweats, or fevers). ${ }^{9}$ The Dynamic International Prognostic Scoring System can be used at any time to estimate prognosis at any time in the disease. ${ }^{10}$ Although the same five items are utilized as the IPSS score, this revised scoring system assigns anemia with two points due to an increased hazard ratio observed with anemia. Patients in this scoring system are separated into low-, intermediate-1-, intermediate-2-, and high-risk categories with survival estimates of 135, 95, 48, and 27 months $(P<0.001)$, respectively. A final scoring system, called Dynamic International Prognostic Scoring System Plus, incorporates mutational status, platelets, and transfusion requirements to further assess the likelihood of leukemia-free survival. ${ }^{11}$ It has been used successfully to predict prognosis on post-ET MF and post-PV MF patients. ${ }^{12}$

Table I Comparison of prognostic scoring methods in myelofibrosis

\begin{tabular}{|c|c|c|c|}
\hline & IPSS 9 & DIPSS $^{10}$ & DIPSS plus ${ }^{\prime \prime}$ \\
\hline Timeframe/assessment & Diagnosis/survival & $\begin{array}{l}\text { Any time point after } \\
\text { diagnosis/survival }\end{array}$ & $\begin{array}{l}\text { Any time point after diagnosis/ } \\
\text { leukemia-free survival }\end{array}$ \\
\hline Age & $\geq 65$ years ( 2 points) & $\geq 65$ years $(\mathrm{I}$ point $)$ & - \\
\hline Leukocytes & $>25 \times 10^{9} / \mathrm{L}(\mathrm{I}$ point $)$ & $>25 \times 10^{9} / L(1$ point $)$ & - \\
\hline Hemoglobin & $<10(\mathrm{I}$ point $)$ & $<10$ (2 points) & - \\
\hline Constitutional symptoms* & Present (I point) versus absent & Present (I point) & - \\
\hline Blasts & $\geq 1 \%$ (I point $)$ & $\geq 1 \%$ (I point $)$ & - \\
\hline \multicolumn{4}{|l|}{ DIPSS score } \\
\hline Low & & & 0 points \\
\hline Intermediate-I & & & I point \\
\hline Intermediate-2 & & & 2 points \\
\hline High & & & 3 points \\
\hline Mutational status & & & Yes (I point) \\
\hline Platelets & & & Yes (I point) \\
\hline Transfusion dependent & & & Yes (I point) \\
\hline \multicolumn{4}{|l|}{ Risk group cutoffs (points) } \\
\hline Low & 0 & 0 & 0 \\
\hline Intermediate-I & I & $\mathrm{I}-2$ & 1 \\
\hline Intermediate-2 & 2 & $3-4$ & $2-3$ \\
\hline High & $\geq 3$ & $5-6$ & $4-6$ \\
\hline
\end{tabular}

Note: *Constitutional symptoms being scored positively if patient had weight loss $>10 \%$ in previous 6 months, night sweats, or fevers.

Abbreviation: DIPSS, Dynamic International Prognostic Scoring System. 


\section{MPN therapies}

Preceding the discovery of JAK2 inhibitors, MPN treatment was limited to targeting disease symptoms and included cytoreductive therapy (hydroxyurea, anagrelide, and phlebotomies), immunomodulatory therapy (thalidomide and lenalidomide), antiplatelet agents (aspirin), androgens (danazol), and steroids. For individuals with medicationrefractory splenomegaly and severe anemia, thrombocytopenia, and constitutional symptoms, splenectomy was considered a final option due to its significant surgical morbidity (31\%) and mortality (9\%). ${ }^{13}$ Stem cell transplantation remains the only potentially curative therapy but is available to a limited population of MPN patients with aggressive disease. ${ }^{14-16}$

\section{Cellular mutations in MF}

The $J A K^{V 617 F}$ mutation is a gain-of-function tyrosine kinase mutation which promotes hematopoietic cell growth and signaling. It is mutated in the majority of MF patients and subsequently represents an attractive target for therapies. The mutation ultimately results in constitutive activation of the kinase domain of JAK1/2 resulting in downstream signal transducer and activator of transcription (STAT) signaling and gene expression that enhances angiogenesis (vascular endothelial growth factor), resists apoptosis (BCL2L1, BIRC5, MCL1), and stimulates cellular growth and regeneration (CCND1). ${ }^{17,18}$ The JAK2 pathway has been shown to be critical in the maturation pathways of both erythropoiesis and thrombopoiesis. ${ }^{19}$ Uniquely, low JAK2 allele burden is associated with poor survival in MF patients, ${ }^{20}$ suggesting that the $J A K^{V 617 F}$ mutation is not disease initiating but instead represents the complex genotypic aberrancies inherent to the condition. Discovery of the JAK2 mutation, JAK2 $2^{V 617 F}$, propelled forward an influx of small-molecule inhibitors specifically targeting the kinase activity. Additional mutations found in MF patients include $M P L, E X H 2, A S X L 1, I D H 1 / 2, T E T 2, C B L$, $I K Z F 1$, and $p 53 .{ }^{21}$

\section{Pharmacokinetics and pharmacodynamics}

3-(4-[7H-pyrrolo[2,3-d]pyrimidin-4-yl]-1H-pyrazol-1-yl)3 -cyclopentylpropanenitrile, later named INCB018424 or ruxolitinib, is a potent orally administered JAK1, JAK2, and JAK3 inhibitor with additional efficacy against the $J A K 2^{\text {V617F }}$ mutation. Results of the metabolism and excretion of ruxolitinib were published by Shilling et al. ${ }^{22}$ Radiolabeled orally administered doses of the drug displayed rapid uptake, metabolism, and $95 \%$ absorption. Time to reach the maximal systemic concentrations was $<1$ hour. The majority of radioactivity was recovered in the form of the parent compound, indicating that the metabolism of ruxolitinib at therapeutic levels of treatment is minimal. The most prevalent metabolite of the compound was 2-hydroxycyclopentyl INCB018424, present in a $16.5 \%$ abundance compared to the unmetabolized compound during day one. Approximately three-quarters of the drug was renally excreted, whereas the rest was removed via fecal elimination.

In 2011, a second study evaluating ruxolitinib metabolism was published by Shi et al, which evaluated the pharmacodynamics and pharmacokinetics among two healthy volunteers. ${ }^{23}$ Doses of 5-200 mg/day were evaluated in both once and twice daily administrations. Maximum tolerated doses were determined to be $25 \mathrm{mg}$ twice daily or $100 \mathrm{mg}$ once daily. Oral availability was excellent despite varying food administration. Plasma half-life was found to be approximately 180 minutes, with elimination of the drug being mainly by liver metabolism with minimal kidney filtration. Additionally, molecular testing was able to indicate higher levels of dephosphorylated STAT3 which correlated with higher levels of ruxolitinib concentration in the blood.

Ruxolitinib is metabolized via the cytochrome P450 3A4 (CYP3A4) pathway, thereby lending itself to many potential drug interactions. In a study conducted by Shi et al, ruxolitinib plasma concentrations were found to increase by $91 \%$ and $27 \%$ when exposed to ketoconazole (strong CYP3A4 inhibitor) and erythromycin (weak CYP3A4 inhibitor), respectively. ${ }^{24}$ Additionally, pretreatment with a CYP3A4 inducer - rifampin - decreased plasma concentrations by $71 \%$ but only decreased overall pharmacodynamics activity by $10 \%$ due to the prevalence of active metabolites. Subsequently, it is recommended that ruxolitinib doses be reduced by half in the setting of strong CYP3A4 inhibitors, with no dose adjustments needed for weak CYP3A4 inhibitors or inducers.

\section{Initial landmark studies}

In November 2010, a landmark Phase I/II joint study by The University of Texas MD Anderson Cancer Center (Houston, TX, USA) and Mayo Clinic (Scottsdale, AZ, USA) demonstrated ruxolitinib's safety and effectiveness among a trial of $153 \mathrm{MF}$ patients. Patients were started on $25 \mathrm{mg}$ twice daily or $25 \mathrm{mg}$ once daily with dose titration $\leq 50 \mathrm{mg}$ twice 
daily or $200 \mathrm{mg}$ once daily. Thrombocytopenia was determined to be the dose-limiting side effect with the maximum tolerated administration being $25 \mathrm{mg}$ twice daily or $100 \mathrm{mg}$ once daily. The majority of participants achieved the primary endpoint of the study, which was $\geq 25 \%$ reduction in spleen length. The effects on spleen size were durable after a mean follow-up of 2 years. ${ }^{25}$

\section{Phase III clinical trials Controlled MF Study with Oral JAK Inhibitor Treatment-I (COMFORT-I)}

COMFORT-I represents the first Phase III trial of ruxolitinib funded by Incyte Corporation ( $\mathrm{N}=309$; Table 2). Conducted among IPSS score intermediate-2- or high-risk MF patients including PMF, post-ET MF, and post-PV MF, the COMFORT-I trial randomized patients in a $1: 1$ ratio to receive ruxolitinib $(n=155)$ or placebo $(n=154)$, with a primary endpoint of $>35 \%$ reduction in spleen volume as assessed by magnetic resonance imaging at 24 weeks. ${ }^{26}$ Secondary endpoints included reduction in symptoms, sustainable drug response, and all-cause mortality. Ruxolitinib-treated patients were administered starting doses of $15 \mathrm{mg}$ twice daily or $20 \mathrm{mg}$ twice daily depending on platelet count. The dose was titrated based on side effects or lack of response. Results of the study indicated that ruxolitinib was successful in reducing spleen size in $41.9 \%$ of patients as compared with $0.7 \%$ in the placebo group at the end of 24 weeks $(P<0.001)$. This effect was durable, with $67 \%$ of patients sustaining a spleen response for $\geq 48$ weeks. Symptom burden, as assessed by the MPN Symptom Assessment Form Total Symptom Score indicated that $45.3 \%$ of patients had a reduction in total score. Reductions in JAK2 allele burden were seen in $10.9 \%$ and $21.5 \%$ of the ruxolitinib-treated patients at weeks 24 and 48, respectively.

Overall rates of adverse events were similar between both ruxolitinib- and placebo-treated groups (11\% of patients receiving ruxolitinib versus $10.6 \%$ of patients receiving placebo). For ruxolitinib-treated patients, grade I/II events included bruising, dizziness, and headaches. Grade III/IV effects included anemia and thrombocytopenia. These hematologic adverse events were noted to occur at a greater frequency in the ruxolitinib-treated group than among placebo-treated patients (thrombocytopenia: 12.9\% ruxolitinib versus $1.3 \%$ placebo; anemia: $45.2 \%$ ruxolitinib versus $19.2 \%$ placebo). Overall, study authors noted a significant survival advantage among patients who received ruxolitinib
Table 2 Comparison of Phase III Controlled Myelofibrosis Study with Oral Janus Kinase Inhibitor Treatment (COMFORT) studies

\begin{tabular}{|c|c|c|}
\hline & COMFORT-| ${ }^{26}$ & COMFORT-III \\
\hline Study design & $\begin{array}{l}\text { Ruxolitinib versus } \\
\text { placebo }(\mathrm{N}=309)\end{array}$ & $\begin{array}{l}\text { Ruxolitinib versus } \\
\text { best available } \\
\text { therapy }(N=219)\end{array}$ \\
\hline Randomization & $\begin{array}{l}\text { I:I (I55 ruxolitinib: } \\
\text { I54 placebo) }\end{array}$ & $\begin{array}{l}2: I \text { (I } 46 \\
\text { ruxolitinib:73 best } \\
\text { available therapy) }\end{array}$ \\
\hline Ruxolitinib dose & $15-20 \mathrm{mg}$ bid & 15-20 mg bid \\
\hline Primary endpoint & $\begin{array}{l}>35 \% \text { reduction in } \\
\text { spleen based on MRI } \\
\text { at } 24 \text { weeks }\end{array}$ & $\begin{array}{l}>35 \% \text { reduction } \\
\text { in spleen volume } \\
\text { (based on } \mathrm{MRI} \text { or } \\
\mathrm{CT} \text { ) at } 48 \text { weeks }\end{array}$ \\
\hline Secondary endpoints & $\begin{array}{l}\text { Duration of spleen } \\
\text { reduction }>35 \% \\
\text { - } \text { Proportion of subjects } \\
\text { with }>50 \% \text { reduction } \\
\text { in MPN-SAF TSS } \\
\text { at week } 24\end{array}$ & $\begin{array}{l}\text { - Overall survival } \\
\text { including } \\
\text { - Progression-free } \\
\text { survival } \\
\text { - Leukemia-free } \\
\text { survival } \\
\text { - > } 35 \% \text { reduction } \\
\text { in spleen volume } \\
\text { at week } 24 \\
\text { - Duration of spleen } \\
\text { reduction }>35 \% \\
\text { - Change in } \\
\text { bone marrow } \\
\text { histomorphology }\end{array}$ \\
\hline
\end{tabular}

Abbreviations: CT, computed tomography; MPN-SAF TSS, Myeloproliferative Neoplasm Symptom Assessment Form Total Symptom Score; MRI, magnetic resonance imaging.

during a 4-month post study follow-up (13 deaths ruxolitinib versus 24 deaths placebo; $P=0.04)$.

\section{COMFORT-II}

COMFORT-II was an open-label investigation sponsored by Novartis AG (Basel, Switzerland) comparing ruxolitinib to best available therapy in patients with IPSS intermediate-2- or high-risk PMF, post-PV MF, and post-ET MF $(\mathrm{N}=219) .{ }^{27}$ Patients were randomized in a $1: 2$ ratio to either receive best available therapy as determined by the investigators $(n=73)$ or to receive $15-20 \mathrm{mg}$ twice per day ruxolitinib $(\mathrm{n}=146)$. Similar to COMFORT-I, the primary endpoint was reduction in spleen volume $(>35 \%$ reduction in volume based on magnetic resonance imaging or computed tomography) assessed at 48 weeks post baseline. In addition to including overall survival similar to COMFORT-I, secondary endpoints were expanded to include leukemia-free and progression-free survival and change in bone marrow morphology. Patients were intended to undergo 48 weeks of treatment, but $25 \%$ of best available therapy-treated patients 
switched to ruxolitinib due to disease progression during this time. In patients treated with best available therapy, $67 \%$ received at least one medication - the most frequent being hydroxyurea (47\%) and steroids (16\%). Symptoms and quality of life were assessed via the European Organization for Research and Treatment of Cancer quality of life questionnaire core model (QLQ-C30) ${ }^{28}$ and the Functional Assessment of Cancer Therapy - Lymphoma scales. ${ }^{29}$

Using intention-to-treat analysis, $28.1 \%$ of patients reached the primary endpoint goal of a spleen volume reduction $\geq 35 \%$ (compared to $0 \%$ of best available therapy patients; $P<0.001$ ). Median time to spleen response was 12.3 weeks. At week 48, 30\% of ruxolitinib-treated patients had experienced progression versus $26 \%$ of patients receiving best available therapy. Six patients treated with ruxolitinib progressed to leukemia or died compared to four patients receiving best available therapy. Analysis of symptoms revealed that ruxolitinib treatment resulted in marked reductions in symptom burden as assessed by both the Functional Assessment of Cancer Therapy - Lymphoma and QLQC30 scales, whereas best available therapy worsened or did not alter symptom burden. Ruxolitinib also appeared to be safe, with the greatest nonhematologic adverse events being diarrhea (23\%) and abdominal pain (3\%). Similar to COMFORT-I, the most serious adverse hematologic events were anemia and thrombocytopenia, which rarely required ruxolitinib discontinuation and were managed by dose titration or transfusions. At the conclusion of the randomization period, $53 \%$ of patients receiving ruxolitinib electively chose to continue ruxolitinib due to its clinical benefit.

\section{Symptom burden relief}

It is estimated that ruxolitinib may be useful in reducing symptom burden among $30 \%$ of MF patients. ${ }^{30}$ In Phase III clinical trials, symptom palliation experienced during ruxolitinib therapy was found to be profound and durable. ${ }^{31}$ Indepth analysis of COMFORT-I data by Verstovsek et al found that the MPN Symptom Assessment Form Total Symptom Score, a ten-item measure assessing the most representative and pertinent of symptoms among MPN patients, indicated consistent improvement in symptom burden regardless of JAK2 mutational status, age ( $>65$ years or $<65$ years), palpable spleen length, baseline hemoglobin $(>10 \mathrm{~g} / \mathrm{dL}$ or $<10 \mathrm{~g} / \mathrm{dL}$ ), or IPSS prognostic group status. ${ }^{32}$ Importantly, $46 \%$ of these patients experienced a $\geq 50 \%$ improvement in symptoms, ${ }^{33}$ compared with only $5.3 \%$ of individuals in the placebo group $(P<0.001)$. Similar findings were found by Harrison et al in an updated analysis of quality of life outcomes from COMFORT-II. ${ }^{34}$ In this study, patients receiving ruxolitinib had persistently improved symptom status assessed by the QLC-C30 and global health status/ health-related quality of life as compared to patients receiving best available alternative therapy at weeks eight and 48 . It has been hypothesized that the majority of these effects stem mainly from its anticytokine effects (eg, suppression of interleukin- 6 and tumor necrosis factor- $\alpha$ ) and, to a lesser extent, nonspecific immunosuppression..$^{35}$

\section{Spleen size reduction}

Splenomegaly stemming from extramedullary hematopoiesis among MF patients is associated with symptoms of early satiety, abdominal discomfort, and pain. ${ }^{36}$ Palliation of massive splenomegaly, traditionally only achievable by means of hydroxyurea, alkylating agents, immunomodulatory drugs, chemotherapy, or splenectomy, may be beneficial for alleviating symptoms. However, no clear improvement exists in overall survival, disease progression, or anemia. ${ }^{37}$ In 2008, Verstovsek et al published results that indicated that administration of ruxolitinib resulted in a rapid decrease in spleen size in $>93 \%$ of MF patients with a mean prestudy spleen size of $>20 \mathrm{~cm}$ (including post-ET, post-PV, and PMF patients). ${ }^{38}$ The decrease in spleen size appeared to be dose dependent, with a reduction of spleen size of $\geq 50 \%$ in $35 \%$ of patients dosed with $10 \mathrm{mg}$ twice daily or $50 \mathrm{mg}$ once daily compared to $59 \%$ of patients dosed with $25 \mathrm{mg}$ twice daily. One year later, updated data released by Verstovsek et al $(\mathrm{N}=309)$ demonstrated that sustainable reductions in spleen volumes were achieved among patients achieving ruxolitinib compared to placebo even after controlling for PMF versus secondary MF, age, risk stratification, presence of $J A K 2^{V 617 F}$ mutational status, anemia status, symptom burden, or spleen size. ${ }^{32}$

\section{Side effects}

Given the critical role of JAK-STAT signaling on erythroid and thrombopoietic production, inhibition of JAK2 secondarily lends itself to key side effects of anemia and thrombocytopenia. This effect was well observed in both COMFORT-I and -II with dose-limiting toxicities being thrombocytopenia. Notably, cytopenias appear to be reversible and dose dependent. ${ }^{25}$ Overall, it appeared that patients started on lower starting doses of ruxolitinib required fewer transfusions (41\% among patients receiving $15 \mathrm{mg}$ twice daily compared to $58 \%$ among patients receiving $20 \mathrm{mg}$ 
Table 3 Novel Janus kinase-2 inhibitor compounds currently under investigation

\begin{tabular}{|c|c|c|c|c|}
\hline \multirow[t]{2}{*}{ JAK2 inhibitor } & \multirow{2}{*}{$\begin{array}{l}\text { Alternative } \\
\text { molecular targets }\end{array}$} & \multicolumn{3}{|c|}{ Phase of investigation } \\
\hline & & Myelofibrosis & Polycythemia vera & $\begin{array}{l}\text { Essential } \\
\text { thrombocythemia }\end{array}$ \\
\hline Ruxolitinib (INCB0I8424) & $\begin{array}{l}\text { JAKI, JAK2, JAK3, } \\
\text { JAK2 }{ }^{\mathrm{V} I 7 F}, \text { STAT3 }\end{array}$ & III/FDA approved & II & II \\
\hline SAR302503 (TGI0I348) & FLT3, Ret & III & I & I \\
\hline CYT387 & JNKI, CDK2 & $1 / I I$ & - & - \\
\hline CEP-70I & FTL3, TrkA & III & II & II \\
\hline AZDI480 & Aurora A, TrkA & $1 / I I$ & - & - \\
\hline Pacritinib (SB|5|8) & FTL3 & II & - & - \\
\hline LY2784544 & Unknown & II & I & 1 \\
\hline
\end{tabular}

Abbreviation: FDA, US Food and Drug Administration.

twice daily). ${ }^{27}$ Upon drug discontinuation, a gradual return of reflexive symptoms may occur. ${ }^{26}$ Generally, these concerning side effects have been successfully managed with dose reduction and tapering.

\section{Future directions}

Applications for ruxolitinib have not been limited to MF. JAK2 mutation and abnormal cytokine expression also play an important role in the pathogenesis of ET, PV, solid tumor malignancies, and immunologic diseases including psoriasis. In patients with hydroxyurea-intolerant or refractory PV and ET, preliminary studies have demonstrated efficacy in improving erythrocytosis, leukocytosis, thrombocytosis, splenomegaly, and phlebotomy independence with ruxolitinib. ${ }^{39}$ Additionally, Phase II studies of ruxolitinib in refractory leukemias including post-MPN acute myeloid leukemia, acute lymphoblastic leukemia, myelodysplastic syndrome, chronic myelomonocytic leukemia, and chronic myelogenous leukemia demonstrated a significant reduction in bone marrow blasts $(<5 \%)$ and decrease in spleen size. ${ }^{40,41}$ Notably, this effect did not seem to correlate with JAK2 mutational status. Additionally, a current Phase II study is underway to investigate the use of ruxolitinib to reducing cachexia and overall mortality in pancreatic cancer. Future studies will likely build on these results to combine ruxolitinib with traditional treatment agents used in advanced stage leukemias or solid tumors malignancies.

\section{Alternative novel MPN therapies}

Many alternative novel compounds are currently in varying stages of investigation for the treatment of MPNs. At least seven JAK inhibitors are currently under investigation, with many of these compounds displaying varying degrees of inhibition to other members of the JAK family, including JAK1, JAK2, JAK3, and tyrosine kinase-2 (Table 3). Along with differences in drug pharmacokinetics and specificity,
JAK inhibitor compounds have shown much variation in hematologic toxicity profiles. Currently, no specific compound is under investigation that specifically targets the $J A K 2^{\text {VG17F }}$ mutation. Pomalidomide is an immunomodulating agent currently in Phase III trials that has been shown to improve anemia with minimal myelosuppression and anemia. ${ }^{42,43}$ Everolimus (RAD001), a potent mammalian target of rapamycin inhibitor, has been successful in preventing in vitro proliferation of $J A K 2^{V 617 F}$ mutated cells and resolving systemic symptoms and pruritus in a cohort of $39 \mathrm{MF}$ patients. ${ }^{44}$ Histone deacetylase inhibitors also represent a growing epigenetic target, with many novel histone deacetylase inhibitors demonstrating the ability to decrease JAK signaling. ${ }^{45}$ Clinical trials of these compounds, including givinostat and panobinostat, are currently ongoing. Additionally, alternative epigenetic agents including the hypomethylating agents azacitidine and decitabine - which function by blocking DNA methyltransferase - have demonstrated promising results in clinical trials.

\section{Conclusion}

The discovery of the JAK mutation influential in the development of MPNs provided scientists with a new therapeutic platform from which to develop targeted treatment strategies. Ruxolitinib represents the first of these compounds and is currently US Food and Drug Administration approved for the treatment of intermediate- and high-risk MF. The pluripotent effects of this therapy stem from its anticytokine effects that result in symptom palliation and reduction of spleen size.

Future challenges in the field of MPN research may be overcome by better understanding of the full spectrum of downstream JAK signaling cascades and their role in providing survival benefit. The majority of preliminary data suggests that if administered in a dose-titrated fashion, ruxolitinib may be safely used in the treatment of MF. More clinical research will be needed before the utility of this 
medication in a routine clinical practice setting can be fully determined.

\section{Disclosure}

The authors report no conflicts of interest in this work.

\section{References}

1. James C, Ugo V, Le Couedic JP, et al. A unique clonal JAK2 mutation leading to constitutive signalling causes polycythaemia vera. Nature. 2005;434(7037):1144-1148.

2. Mesa RA, Silverstein MN, Jacobsen SJ, Wollan PC, Tefferi A. Population-based incidence and survival figures in essential thrombocythemia and agnogenic myeloid metaplasia: an Olmsted County Study, 1976-1995. Am J Hematol. 1999;61(1):10-15.

3. Barbui T, Thiele J, Passamonti F, et al. Survival and disease progression in essential thrombocythemia are significantly influenced by accurate morphologic diagnosis: an international study. J Clin Oncol. 2011;29(23):3179-3184.

4. Tefferi A, Rumi E, Finazzi G, et al. Survival and prognosis among 1263 patients with polycythemia vera: an international study [abstract] Blood. 2011;118(21):277.

5. Tefferi A. JAK inhibitors for myeloproliferative neoplasms: clarifying facts from myths. Blood. 2012;119(12):2721-2730.

6. Tefferi A, Huang J, Schwager S, et al. Validation and comparison of contemporary prognostic models in primary myelofibrosis: analysis based on 334 patients from a single institution. Cancer. 2007; 109(10):2083-2088.

7. Cervantes F, Pereira A, Esteve J, et al. Identification of "short-lived" and "long-lived" patients at presentation of idiopathic myelofibrosis. Br J Haematol. 1997;97(3):635-640.

8. Tefferi A, Pardanani A, Gangat N, et al. Leukemia risk models in primary myelofibrosis: an International Working Group study. Leukemia. 2012;26(6):1439-1441.

9. Cervantes F, Dupriez B, Pereira A, et al. New prognostic scoring system for primary myelofibrosis based on a study of the International Working Group for Myelofibrosis Research and Treatment. Blood. 2009;113(13):2895-2901.

10. Passamonti F, Cervantes F, Vannucchi AM, et al. Dynamic International Prognostic Scoring System (DIPSS) predicts progression to acute myeloid leukemia in primary myelofibrosis. Blood. 2010;116(15):2857-2858.

11. Gangat N, Caramazza D, Vaidya R, et al. DIPSS Plus: a refined Dynamic International Prognostic Scoring System for primary myelofibrosis that incorporates prognostic information from karyotype, platelet count, and transfusion status. J Clin Oncol. 2011;29(4):392-397.

12. Ditschkowski M, Elmaagacli AH, Trenschel R, et al. Dynamic International Prognostic Scoring System scores, pre-transplant therapy and chronic graft-versus-host disease determine outcome after allogeneic hematopoietic stem cell transplantation for myelofibrosis. Haematologica. 2012;97(10):1574-1581.

13. Tefferi A. Myelofibrosis with myeloid metaplasia. N Engl J Med. 2000; 342(17):1255-1265.

14. Mesa RA, Tefferi A. Emerging drugs for the therapy of primary and post essential thrombocythemia, post polycythemia vera myelofibrosis. Expert Opin Emerg Drugs. 2009;14(3):471-479.

15. Mesa RA. New insights into the pathogenesis and treatment of chronic myeloproliferative disorders. Curr Opin Hematol. 2008; 15(2):121-126.

16. Deeg HJ, Gooley TA, Flowers ME, et al. Allogeneic hematopoietic stem cell transplantation for myelofibrosis. Blood. 2003;102(12):3912-3918.

17. Yu H, Jove R. The STATs of cancer - new molecular targets come of age. Nat Rev Cancer. 2004;4(2):97-105.

18. Quintas-Cardama A, Kantarjian H, Cortes J, Verstovsek S. Janus kinase inhibitors for the treatment of myeloproliferative neoplasias and beyond. Nat Rev Drug Discov. 2011;10(2):127-140.
19. Neubauer H, Cumano A, Muller M, Wu H, Huffstadt U, Pfeffer K. Jak2 deficiency defines an essential developmental checkpoint in definitive hematopoiesis. Cell. 1998;93(3):397-409.

20. Tefferi A, Lasho TL, Huang J, et al. Low JAK2V617F allele burden in primary myelofibrosis, compared to either a higher allele burden or unmutated status, is associated with inferior overall and leukemia-free survival. Leukemia. 2008;22(4):756-761.

21. Tefferi A, Jimma T, Sulai NH, et al. IDH mutations in primary myelofibrosis predict leukemic transformation and shortened survival: clinical evidence for leukemogenic collaboration with JAK2V617F. Leukemia. 2012;26(3):475-480.

22. Shilling AD, Nedza FM, Emm T, et al. Metabolism, excretion, and pharmacokinetics of [14C]INCB018424, a selective Janus tyrosine kinase 1/2 inhibitor, in humans. Drug Metab Dispos. 2010;38(11): 2023-2031.

23. Shi JG, Chen X, McGee RF, et al. The pharmacokinetics, pharmacodynamics, and safety of orally dosed INCB018424 phosphate in healthy volunteers. J Clin Pharmacol. 2011;51(12):1644-1654.

24. Shi JG, Chen X, Emm T, et al. The effect of CYP3A4 inhibition or induction on the pharmacokinetics and pharmacodynamics of orally administered ruxolitinib (INCB018424 phosphate) in healthy volunteers. J Clin Pharmacol. 2012;52(6):809-818.

25. Verstovsek S, Kantarjian H, Mesa RA, et al. Safety and efficacy of INCB018424, a JAK1 and JAK2 inhibitor, in myelofibrosis. $N$ Engl $J$ Med. 2010;363(12):1117-1127.

26. Verstovsek S, Mesa RA, Gotlib J, et al. A double-blind, placebocontrolled trial of ruxolitinib for myelofibrosis. N Engl J Med. 2012; 366(9):799-807.

27. Harrison C, Kiladjian JJ, Al-Ali HK, et al. JAK inhibition with ruxolitinib versus best available therapy for myelofibrosis. $N$ Engl J Med. 2012;366(9):787-798.

28. Aaronson NK, Ahmedzai S, Bergman B, et al. The European Organization for Research and Treatment of Cancer QLQ-C30: a quality-of-life instrument for use in international clinical trials in oncology. $J$ Natl Cancer Inst. 1993;85(5):365-376.

29. Cella DF, Tulsky DS, Gray G, et al. The Functional Assessment of Cancer Therapy scale: development and validation of the general measure. J Clin Oncol. 1993;11(3):570-579.

30. Tefferi A, Lasho TL, Jimma T, et al. One thousand patients with primary myelofibrosis: the Mayo Clinic experience. Mayo Clin Proc. 2012;87(1):25-33.

31. Tefferi A, Litzow MR, Pardanani A. Long-term outcome of treatment with ruxolitinib in myelofibrosis. $N$ Engl J Med. 2011; 365(15):1455-1457.

32. Verstovsek S, Mesa RA, Gotlib JR, et al. Consistent benefit of ruxolitinib over placebo in spleen volume reduction and symptom improvement across subgroups and overall survival advantage: results from COMFORT-I [abstract]. Blood. 2011;118(21):278.

33. Ostojic A, Vrhovac R, Verstovsek S. Ruxolitinib: a new JAK1/2 inhibitor that offers promising options for treatment of myelofibrosis. Future Oncol. 2011;7(9):1035-1043.

34. Harrison CN, Kiladjian JJ, Al-Ali HK, et al. Health-related quality of life and symptoms in myelofibrosis patients treated with ruxolitinib versus best available therapy [abstract]. Blood. 2011;118(21):795.

35. Tefferi A. Challenges facing JAK inhibitor therapy for myeloproliferative neoplasms. N Engl J Med. 2012;366(9):844-846.

36. Scherber R, Dueck AC, Johansson P, et al. The Myeloproliferative Neoplasm Symptom Assessment Form (MPN-SAF): international prospective validation and reliability trial in 402 patients. Blood. 2011;118(2):401-408.

37. Mesa RA. How I treat symptomatic splenomegaly in patients with myelofibrosis. Blood. 2009;113(22):5394-5400.

38. Verstovsek S, Kantarjian H, Pardanani A, et al. The JAK inhibitor, INCB018424, demonstrates durable and marked clinical responses in primary myelofibrosis (PMF) and post-polycythemia/essential thrombocythemia myelofibrosis (post PV/ET-MF) [abstract]. Blood. 2008;112:1762a. 
39. Verstovsek S, Passamonti F, Rambaldi A, et al. Durable responses with the JAK1/JAK2 inhibitor, INCB018424, in patients with polycythemia vera (PV) and essential thrombocythemia (ET) refractory or intolerant to hydroxyurea (HU) [abstract]. Blood. 2010;116(21):313.

40. Eghtedar A, Verstovsek S, Cortes J, et al. Phase II study of the JAK2 inhibitor, INCB018424, in patients with refractory leukemias including post-myeloproliferative disorder (MPD) acute myeloid leukemia (sAML) [abstract]. Blood. 2010;116(21):509.

41. Ravandi F, Verstovsek S, Eztrov Z, et al. Significant activity of the JAK2 inhibitor, INCB018424 in patients with secondary, post-myeloproliferative disorder (MPD) acute myeloid leukemia (sAML): results of an exploratory phase II study [abstract]. Blood. 2009; 114(22):631.
42. Tefferi A, Verstovsek S, Barosi G, et al. Pomalidomide is active in the treatment of anemia associated with myelofibrosis. J Clin Oncol. 2009;27(27):4563-4569.

43. Begna KH, Mesa RA, Pardanani A, et al. A phase-2 trial of low-dose pomalidomide in myelofibrosis. Leukemia. 2011;25(2):301-304.

44. Guglielmelli P, Barosi G, Rambaldi A, et al. Safety and efficacy of everolimus, a mTOR inhibitor, as single agent in a phase $1 / 2$ study in patients with myelofibrosis. Blood. 2011;118(8):2069-2076.

45. Vannucchi AM, Guglielmelli P, Rambaldi A, Bogani C, Barbui T. Epigenetic therapy in myeloproliferative neoplasms: evidence and perspectives. J Cell Mol Med. 2009;13(8A):1437-1450.

\section{Publish your work in this journal}

Blood and Lymphatic Cancer: Targets and Therapy is an international, peer-reviewed, open access journal focusing on blood and lymphatic cancer research, identification of therapeutic targets and the optimal use of preventative and integrated treatment interventions to achieve improved outcomes, enhanced survival and quality of life for the cancer patient. The manuscript management system is completely online and includes a very quick and fair peer-review system. Visit http://www.dovepress.com/testimonials.php to read real quotes from published authors. 